\title{
LE ORAZIONI NEL LIBER MAIORICHINUS
}

\section{Prayers in the Liber Maiorichinus}

\author{
Prof. Dr. Alberto Cotza \\ (Università di Pisa - Dipartimento di Civiltè e Forme del Sapere) \\ ORCID: https://orcid.org/0000-0001-7834-8013 \\ E-mail: alberto.cotza@cfs.unipi.it
}

Recebido em: 05/06/2020

Aprovado em: 20/07/2020

\begin{abstract}
Riassunto: Il Liber Maiorichinus, un poema epico redatto a Pisa probabilmente negli anni '20 del XII secolo per raccontare la guerra dei Pisani e dei loro alleati contro i Musulmani delle Baleari (1113-1115), è una delle prime opere della storiografia italiana cittadina del periodo a riportare, nella forma del discorso diretto, le orazioni pubbliche tenute sul campo di battaglia. A questo ricco corpus di discorsi non è stata prestata la dovuta attenzione. I discorsi pubblici nel Liber sono, invece, una fonte fondamentale per indagare il rapporto tra oratoria e società cittadina nella prima metà del XII secolo, quando scarse, rispetto alla fase comunale matura, sono le testimonianze che consentano di approfondire questo tema. Chi parla e come nel Liber Maiorichinus? Per quale scopo si tiene un'orazione? A quali modelli aderisce? Il contributo cercherà di offrire una prima risposta a queste domande.
\end{abstract}

Parole chiave: Liber Maiorichinus; orazioni pubbliche; guerra.

Abstract: The Liber Maiorichinus is an epic poem dealing with the history of the Balearic war, which the Pisans and other Christians conducted against the Muslims in Ibiza, Minorca, and Mallorca (1113-1115). The oeuvre is not just the most detailed, nearly contemporary account of what happened during the war. It is also a source to study how local élites acted in the public scenario. In this regard, public speeches have primary importance. Though neglected by historians, those speeches are a key element for understanding the use of rhetoric in the 12th century Pisan society. Who pronounces those speeches, and why? Are there comparable models? Are those speeches that we know from the Liber part of a formalized practice of public speaking? This paper attempts to answer those questions.

Keywords: Liber Maiorichinus; public speeches; war. 


\section{Le orazioni nel Liber Maiorichinus}

1. Il Liber Maiorichinus è un poema epico in esametri che racconta, in otto libri da circa 500 versi ciascuno, le vicende dell'assedio cristiano alle isole Baleari (1113-1115) dal punto di vista dei Pisani, uno dei numerosi contingenti militari protagonisti della guerra. ${ }^{1}$ Anonimo, redatto attorno alla metà degli anni '20 del XII secolo, quindi un po' di tempo dopo i fatti narrati, l'opera è importante perché ci consente di conoscere, insieme a poche altre fonti, pisane e no, lo svolgimento della guerra, anche se filtrato dalle sole parole dei vincitori. ${ }^{2}$ Quest'ultimo è stato l'uso della fonte che fino ad ora è prevalso tra gli studiosi: un serbatoio di informazioni da combinare con notizie di altre fonti per la ricostruzione di un quadro complessivo dell'impresa balearica. Si tratta di una prospettiva di storia evenemenziale, viziata dalla parzialità delle informazioni di partenza. Ricostruire la storia degli eventi non è un compito dal quale, in termini generali, bisogna sottrarsi; per essere assolto, tuttavia, occorre che l'insieme di discorsi, linguaggi, interpretazioni all'interno dei quali è inserita la narrazione dei fatti sia chiara. A ciò si aggiunga che non tutti i contenuti del Liber sono utilizzabili ai fini della storia evenemenziale. All'autore del poema non premeva solo di raccontare la versione dei fatti che più glorificasse il contributo pisano alla sconfitta dei "Saraceni". La sua intenzione era anche di mettere in scena le virtù dei Pisani: virtù soprattutto politiche, nel senso più ampio del termine, da intendere come un insieme complesso di arti del governo della città atte a garantire stabilità interna e capacità di coordinamento della forza militare della città - nel caso specifico del Liber Maiorichinus contro il pericolo rappresentato dai Musulmani.

Non ci troviamo di fronte a un elenco di fatti ed eventi, una cronaca compilativa, ma a un testo più complesso. Il poema potrebbe essere inteso come una sorta di speculum civitatis: vi ritroviamo la rappresentazione di una città ideale, le cui caratteristiche andrebbero commisurate, nel loro grado di affidabilità storica, col raffronto con altre fonti, non solo narrative. Tuttavia, anche di per se stessa, questa rappresentazione ideale è significativa: ci consente di apprezzare i modelli politici e sociali che le élites intellettuali cittadine - in questo caso, $\mathrm{i}$ canonici della cattedrale tra $\mathrm{i}$ quali va collocato l'anonimo autore del poema - prendevano a riferimento nel tentativo di descrivere come funzionasse una città agli inizi del XII secolo, quando a Pisa e, più in generale nelle città dell'Italia centrosettentrionale, nuove forme di governo comunali si stavano affermando sullo scenario politico. In questo breve saggio, guarderò con particolare attenzione a un aspetto di questo problema, quello delle orazioni pubbliche tenute nel contesto della guerra balearica. Si tratta di una delle poche fonti che ci consente di osservare da vicino questo fenomeno. Numerose sono le orazioni riportate nella forma del discorso diretto nel Liber Maiorichinus; altre volte le orazioni vengono solo citate o riportate in forma indiretta. Si intuisce comunque, anche a una prima lettura, che costituiscano uno degli assi strutturali del racconto. Quali sono le loro caratteristiche principali e cosa ci dicono delle élite cittadine comunali dei primi decenni del XII secolo?

2. Partiamo da qualche dato numerico. Nel Liber si contano in tutto 32 orazioni tra quelle riportate nella forma del discorso diretto e le menzioni parziali e indirette. Un numero elevato: se volessimo fare una media dei discorsi tenuti nel Liber, dovremmo dire che ci sono quattro orazioni per libro. Considerando circa 500 versi per libro, 
sarebbe come dire che ogni 100 versi circa - lo spazio di una scena - vi sia l'intervallo di un'orazione. Tuttavia, un calcolo di questo tipo non rende ragione della reale distribuzione delle orazioni nell'opera, che è estremamente diseguale. In alcuni libri si contano molte più orazioni della media, mentre in alcuni libri non ve ne è nessuna: nel primo se ne contano otto, mentre nel quinto libro non ve ne è nessuna. Le ragioni di questa distribuzione diseguale vanno ricercate nella funzione che è attribuita ai discorsi pubblici nel Liber Maiorichinus: solo in alcuni momenti, personaggi particolarmente eminenti - come vedremo - ricorrono ai discorsi pubblici; in altri momenti, invece, non è necessario che siano tenuti discorsi collettivi. Possiamo notare, in particolare, che i libri dedicati al racconto degli assedi - come il quarto, destinato alla narrazione della conquista di Ibiza, il quinto, in cui si racconta l'approdo a Maiorca e i primi combattimenti contro i locali, o l'ottavo dedicato alla conquista della stessa isola - sono i più poveri di orazioni; al contrario, i libri dedicati alla preparazione delle imprese come, ad esempio, il primo, dedicato ai preparativi per la partenza o il settimo, nel quale vengono descritti i giorni precedenti all'assedio finale di Maiorca - sono intessuti di orazioni. Questi libri mettono in scena i momenti decisionali, all'interno dei quali assumono un ruolo primario i discorsi pubblici. È come se la vita della militia pisana sul campo di battaglia fosse divisa tra la parola e i fatti, tra i discorsi e la guerra. Quando si parla, non si combatte, e viceversa.

Analizziamo più da vicino il lessico dedicato a questi momenti. Particolarmente significativo è che alla descrizione dei momenti preparatori delle orazioni sia dedicato un lessico specifico, che lascia intendere che i discorsi tenuti sul campo non fossero "improvvisati" ma fossero inseriti in un contesto pubblico e simbolico formalizzato o, se si vuole, ritualizzato. Se prendiamo, ad esempio, uno dei discorsi menzionati in forma indiretta nel sesto libro, possiamo cogliere con chiarezza questo aspetto. ${ }^{3}$ Durante una delle notti che precedettero l'assalto finale, raccontato con più dettagli nel settimo e soprattutto nell'ottavo libro, i Pisani furono attaccati di sorpresa dai loro nemici, che riuscirono a bruciare due delle torri lignee che erano state allestite in vista dell'ultima battaglia. I Pisani che erano stati posti a guardia si erano addormentati e non avevano svolto onorevolmente il loro compito, mettendo a rischio la riuscita dell'impresa. La mattina successiva era il momento di capire cosa fosse successo, come comportarsi con i colpevoli e come rimediare al danno subito. Ecco che, allora, i «patres» - anche così vengono chiamati i consoli con un termine di sapore antico - «danno ben pronto il segno della concione». Fu così che «all'adunata («ad cetum») giunge ormai da ogni parte tutta la schiera. Azzo primo si lagna («queri»), ma tutta l'assemblea lo segue mentre con brevi parole narra i singoli eventi, alcuni chiamando ignavi o spregevoli e traditori del giuramento di fraterno amore».

I termini usati per descrivere il contesto nel quale Azzo aveva preso la parola «concio» - ricorre almeno un'altra volta nel Liber e numerose volte si incontra anche il termine «cetum», ad indicare la riunione della «militia» in un luogo comune, sempre in relazione al momento in cui era necessario discutere e prendere la parola in pubblico. ${ }^{4}$ Un richiamo noto, poi, doveva essere quello usato dai consoli per convocare l'assemblea. Ma da rilevare in questo passo è soprattutto il verbo usato per riferirsi all'orazione di Azzo, il verbo «queror» che rimanda alla sfera semantica del lamento. Insieme a questo verbo, troviamo spesso nel Liber il sostantivo «querela», sempre riferito a lagnanze esposte in pubblico. Se ne contano almeno una trentina di attestazioni; è uno dei termini chiave per capire il carattere delle orazioni pubbliche nel Liber. Anche tutti i passi nei quali compare questo termine potrebbero essere considerati 
come menzioni indirette di orazioni. In generale, è un termine che fa riferimento alla lamentela pubblica di un gruppo di persone contro qualcun altro. Per noi è significativo perché, al di fuori del contesto letterario del Liber, il verbo «quero» e i termini ad esso associabili sono caratteristici di una tipologia documentaria particolarmente diffusa nel XII secolo. Si tratta delle cosiddette "carte di querela", documenti in via di formalizzazione tra XI e XII secolo, atta ad accogliere le «lamentationes» di soggetti contro altri soggetti, che potevano poi essere discusse e confrontate in sede giudiziaria. ${ }^{5}$ Solitamente queste carte erano aperte dal verbo «conqueror», a cui seguiva l'elenco delle malefatte di coloro contro i quali si muoveva in giudizio. Sembra che, nel passo del Liber citato e nei numerosi altri passi in cui ricorrono le parole dello stesso campo semantico, si faccia riferimento a una tipologia di discorso simile. In effetti, per Azzo, seguito dalla folla che lo ascoltava, si trattava di prendere la parola per capire chi fossero i responsabili dell'atto che aveva causato l'attacco nemico perché poi gli stessi personaggi fossero puniti: una sorta di "processo". Notiamo, in questo senso, un'intersezione tra il lessico "aulico" del Liber e il lessico documentario più diffuso.

Il verbo «queror» e i sostantivi associati sono prevalenti, ma non sono gli unici usati per riferirsi ai discorsi. Frequente - almeno dieci attestazioni - è il termine «sermo». Potrebbe trattarsi di una tipologia di discorso diversa da quella indicata con il termine «querela». Talvolta è accompagnato da aggettivi che lo qualificano e che possono riferirsi a modalità allocutorie differenti: «brevis sermo», «cautus sermo», «planus sermo», «patulus sermo». Contesti diversi richiedevano che il tono dei discorsi, la loro lunghezza e la loro complessità fossero differenti. Non sappiamo se tali differenze fossero in qualche modo codificate, se fossero oggetto di una trasmissione di conoscenze o se, piuttosto, fosse la pratica più che la teoria a regolare il modo in cui si doveva parlare in pubblico rivolgendosi a interlocutori e momenti diversi.

$\mathrm{Ma}$, a parte le forme e i modi concreti nei quali si svolgevano le orazioni, che è difficile conoscere nel dettaglio, sembra comunque di poter dire che l'uso di un lessico specifico in riferimento alle occasioni nelle quali si prendeva la parola rimandi alla formalizzazione delle pratiche della discussione pubblica. Sebbene non abbiamo testimonianze dirette sulle modalità di trasmissione e formalizzazione delle pratiche dell'oratoria, si può dire comunque che doveva esistere, nella Pisa dei primi decenni del XII secolo, una tendenza alla regolamentazione di questa forma di espressione pubblica. La stessa conclusione si può trarre, per contrasto, guardando al modo in cui vengono etichettati i discorsi di individui che nel Liber prendono la parola al di fuori delle regole che normavano le orazioni sul campo di battaglia. Particolarmente interessante, da questo punto di vista, è il termine «murmur» e il verbo «murmurare», del quale si contano almeno cinque attestazioni. È un termine con una connotazione negativa, associato ai discorsi di persone che mettevano a repentaglio la buona riuscita dell'impresa.

Prendiamo, ad esempio, un caso significativo descritto nel primo libro. Partita la flotta alla volta delle Baleari, alcuni membri dell'esercito cominciano a nutrire qualche dubbio sull'opportunità dell'impresa. Il mare agitato, il timore della guerra, le possibili sventure sconfortano gli animi. Ne nascono discussioni. Così descrive l'autore questi momenti di dissenso interno: «Frattanto il volgo, deposto l'estremo timore del mare, mormora e turpemente anela al patrio lido». ${ }^{6}$ Le rimostranze del «vulgus» vengono definite «mormorii» ma siamo propensi a credere che si trattasse di veri e propri contrasti interni, sfociati in discussioni pubbliche. Lo sguardo dell'autore, però, volto ad esaltare la concordia dell'esercito nel raggiungimento dell'obiettivo, minimizza questi 
momenti. La reazione ai "mormorii" comportò, comunque, l'intervento di uno dei membri più illustri degli alleati dei Pisani: il lucchese Fraolmo. Costui dice chiaramente, nel discorso fatto per osteggiare coloro che volevano tornare in patria, chi fosse a parlare in quel modo: «Forse vi pentite, ignavi, del nobile cimento? Perché la voce di costoro contamina la luce dell'aria? Certo si rammarica d'essere giunto chi soleva coltivare i campi, sempre piegato sugli aratri, e tutto il tempo trascorreva rivoltando zolle; e adesso, non potendo spargere nel solco le sementi o piegarsi sul mosto calpestato coi piedi, notte e giorno lamenta (queruntur) questi indugi e questi eventi minacciando, forse, di tornare alla propria casa. Ah, vili coloro la cui vita miseranda è affatto inutile al popolo qui radunato!». Si trattava del «vulgus», degli «ignavi», di coloro «qui rura solebant vertere», insomma di contadini. Persone in cerca di fortuna, pronti a sfruttare un'occasione di bottino che poteva essere un'irripetibile opportunità di ascesa sociale. In ogni caso, un settore della società differente da quello dei «milites» che guidavano l'impresa, abituati a una vita diversa. Diversamente dai cavalieri, poi, erano anche poco abituati a parlare: lo facevano in modo disordinato e caotico. In un altro passo del secondo libro, la «rustica turba» - così come viene chiamato l'insieme di coloro che non erano considerati parte della «militia» - si dice che parli «in preda all'ira, secondo il suo costume (more suo), agitata e in tumulto». ${ }^{7} \mathrm{Da}$ questi passi si può trarre l'idea che il modo di parlare adatto a esprimere opinioni differenti nel contesto pubblico delle adunanze militari richiedesse alcune conoscenze e capacità acquisibili col tempo. Venivano trasmesse, forse informalmente, anche grazie alla frequentazione di un universo sociale nel quale parlare in pubblico stava diventando uno degli elementi caratteristici per governare. Non tutti potevano prendere la parola in pubblico allo stesso modo.

3. Guardiamo ora, in modo più ravvicinato, ai protagonisti delle orazioni. Chi parla nel Liber Maiorichinus? Una distinzione fondamentale corre tra ecclesiastici e laici. I primi, rappresentanti dal vescovo Pietro, dal gruppo di canonici della cattedrale che lo seguiva e da pochi altri esponenti come il cardinale Bosone e l'arcivescovo di Cagliari, Guglielmo, hanno un ruolo primario nei discorsi pubblici - pur essendo numericamente inferiori rispetto al gruppo dei laici. Potrebbe trattarsi di una distinzione accentuata dall'autore del Liber, che mette in rilievo la preminenza della chiesa nell'organizzazione della società pisana. Prendiamo, a titolo d'esempio, una scena del secondo libro dalla quale traspare con chiarezza questa forma di rappresentazione dell'oratoria. Si tratta, ancora una volta, di affrontare le difficoltà poste da alleati reticenti alla prosecuzione dell'impresa: «Mentre le schiere indugiano vagando per deserti campi, si leva un mormorio; le torme lucchesi, infine, che sanno sopportare meno il mare e i flutti, troppo intese a trattare gli alleati come schiavi, ogni giorno riempiono le folle di lamenti». ${ }^{8}$ Seguono tre discorsi, disposti in ordine gerarchico.

Il primo è quello di Ugo, arciprete della canonica, che esorta gli alleati non intenzionati a proseguire, a lasciare il campo - anche perché ciò non poteva essere impedito da nessuno. I Pisani potevano vietare solo ai Pisani di non proseguire l'impresa ma non al resto del gruppo. I Lucchesi, d'altra parte, si erano uniti all'esercito volontariamente, senza la costrizione di nessuno. Il secondo discorso è di Guido, un altro canonico della cattedrale. Anche il suo discorso ha al centro la volontarietà della partecipazione lucchese e l'assenza di costrizioni. Caratteristico della sua orazione è il paragone tra i Lucchesi e gli Ebrei. Questi ultimi «avrebbero desiderato sopportare il giogo del crudele tiranno e rimanere schiavi, piuttosto che bandire il vizio della gola o rinunciare al cibo. Di quel popolo, voi serbate i costumi, l'animo e i modi». Guido 
rimprovera agli alleati di essere poco disposti a fare sacrifici e di preferire le comodità della patria alle difficoltà di un'impresa. Dopo il discorso di Guido, l'autore registra l'intervento di Guglielmo di Montpellier, disposto a rimpiazzare ogni lucchese con quattro dei suoi. Chiude la serie di discorsi l'intervento di Enrico di Guinizzo, dell'importante famiglia dei Sismondi. La sua orazione, a differenza di quelle che lo avevano preceduto, non viene riportata nella forma del discorso diretto. L'autore registra il modo in cui Enrico aveva parlato, in piedi, e il contenuto del suo discorso: un'esortazione a proseguire la guerra, seguita dall'incitamento a giurare tutti insieme.

Come si vede, l'autore dà rilievo primario ai discorsi degli ecclesiastici, mettendo in secondo piano quelli dei laici. È una strategia che egli persegue anche attraverso l'accentuazione delle differenze formali tra i discorsi degli uni e degli altri. Ciò è particolarmente evidente nel caso di una delle orazioni del vescovo Pietro. ${ }^{9}$ Siamo nel primo libro e il presule richiama l'attenzione dei militi sull'importanza della concordia, nel tentativo di sedare i dissidi che capitavano di frequente. «Odio, discordia, livore e ira» vengono considerati da Pietro come peccati che discendono direttamente da Adamo e dei quali i Pisani, soprattutto in un'impresa così importante, non dovevano macchiarsi. Per noi è significativo che in questo discorso, che si occupa di uno dei problemi principali per l'organizzazione dell'impresa, sia riportato in esametri caudati con rima bisillabica, in una struttura quindi che accentua la raffinatezza formale del testo e la sua ritmicità, con l'intenzione di renderlo più piacevole per il lettore. Si trattava anche di mettere in rilievo la capacità del vescovo - a differenza di tutti gli altri che prendono la parola - di usare uno stile più elaborato, riflesso di una formazione culturale più elevata $\mathrm{e}$, in modo indiretto, di una posizione politica eminente. Vediamo un esempio: «Nascitur hinc odium, discordia livor et ira:/ Ista movere queunt hominum precordia dira;/ Non valet esse bonus vitiis qui subiacet istis; / Vincere nemo potest quisquis superatus ab ipsis». ${ }^{10}$

Per quanto riguarda, invece, i laici, a prendere la parola è un gruppetto di persone ristretto: Ildebrando di Rolando, console (Orlandi), Azzo di Marignano, console (Marignani), Fraolmo di Lucca, Enrico di Guinizzo, console (Sismondi), il conte di Ampurias, Nazaredeolo, re di Maiorca, Lamberto di Uberto, console (De Curte), il duca di Catalogna, Carlo (?), Pietro di Albizo (Casapieri). A parte i non Pisani, si tratta degli esponenti di alcune delle principali famiglie pisane che in quegli anni reggeva il consolato. ${ }^{11}$ Come si vede, la maggior parte dei Pisani citati sono consoli. L'unico a prendere la parola che nel biennio balearico non ricopriva la carica è Pietro di Albizo e l'ignoto Carlo, il personaggio scelto dai Pisani per l'ultima ambasceria al re di Maiorca. Si trattava comunque di personaggi dello stesso rilievo sociale e politico. È evidente, da quanto si evince dal Liber, che la capacità di saper parlare in pubblico fosse considerata una condizione fondamentale per governare, uno dei modi attraverso il quale i consoli esercitavano il potere.

Particolarmente interessante, da questo punto di vista, è il discorso tenuto insieme da Enrico e Azzo nel settimo libro. I due consoli esprimono riprovazione nei confronti degli alleati catalani, che avevano portato avanti le trattative con Nazaredeolo, al punto da arrivare quasi a concludere un accordo. È particolarmente interessante che i consoli non tengano due orazioni separate, ma che insieme - non sappiamo in che modo: il Liber dice che «his respondentes aiunt Henricus et Azo» - pronuncino lo stesso discorso, forse allo stesso momento (almeno secondo quanto vuole farci credere il Liber). Ciò doveva accrescere l'autorevolezza di quanto detto. Ma, allo stesso tempo, ci informa anche in modo indiretto delle forme di elaborazione dei discorsi pronunciati dai 
consoli, quando si rivolgevano ad autorità esterne alla città: doveva esistere una consultazione preliminare su cosa dire e come dirlo.

Non sappiamo - ancora una volta, procediamo in modo indiziario - quali fossero le procedure che regolavano consultazioni di questo tipo. Possiamo dire che Enrico e Azzo, scelti per questo importante discorso che aveva richiesto la presenza di entrambi di fronte ai Catalani, avevano già pronunciato altri discorsi di fronte ai Pisani e agli alleati. Abbiamo già incontrato Enrico nelle pagine precedenti; Azzo, invece, menzionato per la prima volta nell'elenco dei consoli, viene definito poco dopo, nel momento in cui pronuncia un'orazione, «per la facondia grato al popolo e illustre». Ne vengono messe in particolare rilievo le doti oratorie. Non sembra un caso, allora, che proprio Enrico e Azzo fossero stati scelti per pronunciare quel discorso di fronte agli alleati. Sembra che alcuni personaggi fossero più adatti di altri a parlare in pubblico. Si potrebbe pensare che avessero seguito un percorso di formazione migliore, non sappiamo in quali luoghi, secondo quali modalità, incentrato su quali discipline. Tuttavia, possiamo notare qualche particolarità: dallo studio di Rossi sulle sottoscrizioni nei documenti pisani dei secoli XI-XII, gli esponenti della famiglia Sismondi, ai quali apparteneva Enrico, sono risultati, tra le famiglie dell'élite di governo, tra coloro che avevano investito di più sulla formazione grafica e, più in generale, culturale. Scrive Rossi: «senza volere in alcun modo proporre una relazione scrittura-potere che, del resto, non emerge affatto dai documenti in maniera univoca, non mi sembra trascurabile il peso che la cultura grafica ebbe nell'affermazione sociale di questi personaggi». ${ }^{12}$ Non è difficile immaginare che, a una formazione grafica di alto livello, potesse accompagnarsi una formazione retorica, che nei primi decenni del XII secolo era, come abbiamo visto, uno degli elementi caratteristici delle élite cittadine. In ogni caso, a parte la particolarità formale dell'orazione del vescovo di cui abbiamo detto sopra - tra l'altro occorre notare che solo una tra le orazioni di Pietro presenta quella peculiarità - non esistono differenze sostanziali tra le orazioni degli ecclesiastici e quelle dei laici. Esistono domini tematici differenti: mentre i discorsi di Pietro e dei canonici tendono a mettere in rilievo lo scopo pio dell'impresa e a esortare i militi alla concordia, quelli dei laici sono più esortazioni alla guerra e richiami alla necessità di combattere nei confronti degli alleati.

Al di fuori di questo gruppo di persone, non c'è nessun altro che prenda la parola. Esisteva la possibilità, per chi non avesse lo stesso rilievo sociale di questi personaggi, di approvare con acclamazioni collettive i discorsi dei personaggi più importanti. Esistevano, poi, anche orazioni collettive, come quella pronunciata dalla folla festante dopo la sottomissione di Ibiza e la partenza verso Maiorca: «A Maiorca! - grida la folla - La stella chiomata esorta a correre ad essa, a volgere con forza! Ci è dato di soggiogare il regno balearico: vedete i raggi della stella caduti a Maiorca». ${ }^{13}$ Si tratta, come si vede, non di una semplice approvazione ma di un vero e proprio discorso di tutta la folla. Alto è il grado di rielaborazione letteraria del Liber, ma non è da sottovalutare che vi sia un contenuto di verità quando nell'opera vengono riportate $o$ solo menzionate esortazioni di questo tipo. Si potrebbe trattare di discorsi elaborati da un ristretto gruppo di persone e poi diffusi perché venissero cantati, recitati collettivamente per rinfrancare gli spiriti e spingere alla battaglia. Siamo su un livello diverso e più elementare rispetto alle orazioni tenute dai personaggi più eminenti.

4. Si pone il problema della veridicità delle orazioni riportate nel Liber Maiorichinus. È difficile immaginare che il vescovo, gli ecclesiastici e i consoli pronunciassero le loro orazioni in esametri, così come sono riportate nel Liber. Tuttavia, 
ciò non deve condannare le orazioni del Liber a un giudizio di falsità. Inoltre, porre la questione nei termini di verità/falsità non è il modo migliore per affrontare il problema. Si tratta di capire, al contrario, di quale aspetto della realtà fossero riflesso le orazioni nella versione indubbiamente alterata che troviamo nel poema, rinunciando a considerarle o solo vere o solo fittizie. Non si può dire che non abbiano un contenuto di realtà, così come non si può dire - al contrario - che siano specchio fedele di come si siano tenute le orazioni sul campo. Trovare una risposta a questa domanda è comunque complesso. Non abbiamo metri di paragone nella coeva produzione documentaria e narrativa che ci consenta di capire in quali termini le orazioni del Liber siano reali. Tuttavia, possiamo almeno provare a capire se nella relativamente ricca produzione storiografica precedente al Liber Maiorichinus le orazioni avessero lo stesso rilievo che hanno in quest'opera. Una risposta negativa consentirebbe di cogliere nella composizione del poema balearico una mutazione nelle forme di rappresentazione del fenomeno, specchio di una sua maggiore rilevanza sociale. Possiamo prendere in considerazione, a questo scopo, due opere importanti della produzione narrativa pisana: il carme sull'impresa contro i Saraceni del 1087 e i Gesta Triumphalia per Pisanos facta.

La prima opera, un poema di 290 settenari composto poco dopo i fatti raccontati, narra l'assalto dell'esercito cristiano composto da Pisani, Genova, Amalfitani alla città di al-Mahdia (nell'attuale Tunisia) nell'agosto del $1087 .{ }^{14}$ In quest'opera è riportato un solo discorso, quello del vescovo Benedetto di Modena, un presule filogregoriano posto a capo dell'impresa dalla marchesa di Tuscia, Matilde, e dal gruppo di cittadini filomatildici. La sua, una lunga allocuzione dedicata a persuadere l'esercito a non stringere patti con Timino e a proseguire la guerra, è l'unica riportata nella forma del discorso diretto; le discussioni, che pure dovevano esservi state tra il vescovo e i laici chiamati al «concilium» - così come viene definita la riunione convocata da Benedetto per discutere della prosecuzione dell'impresa - non vengono menzionati. L'autore del poema dà spazio al discorso del vescovo, l'unico che era stato in grado di condurre l'impresa a un felice esito, evitando che l'esercito fosse distratto dalle ricchezze promesse da Timino in cambio della fine della guerra.

Una situazione diversa è quella dei Gesta Triumphalia per Pisanos facta, un'opera in prosa la cui stesura definitiva è collocabile tra il 1119 e il 1120 a memoria di tutte le gloriose imprese dei Pisani, a partire dalla partecipazione alla prima crociata. ${ }^{15}$ In quest'opera non sono riportate orazioni nella forma del discorso diretto, mentre vengono menzionate occasioni durante la quali vi furono certamente discussioni. Un esempio significativo è il «conventus» che si tenne poco prima della conclusione della guerra balearica, quando i Catalani stavano per stringere un patto con Nazaredeolo. È lo stesso episodio che abbiamo visto descritto nel Liber Maiorichinus, dove viene registrato l'intervento di Enrico e Azzo. Nei Gesta la discussione non viene menzionata (anche se il termine «conventus» la lascia presupporre), mentre vengono raccontati in modo molto più asciutto $\mathrm{i}$ fatti. In questo caso la narrazione si presenta come una compilazione di eventi, poco attenta alla concretezza della situazione nella quale si erano prese le decisioni.

Rispetto al Liber Maiorichinus, queste fonti, che in alcuni casi trattano gli stessi eventi - è il caso dei Gesta rispetto al Liber - riservano molto meno spazio alla rappresentazione delle orazioni. Se confrontiamo poi le due fonti tipologicamente più vicine, perché entrambe scritte in versi e di taglio monografico, il carme sull'impresa del 1087 e il Liber Maiorichinus - possiamo notare un notevole scarto: nel poema dedicato all'impresa balearica non è solo il vescovo a prendere la parola ma molto 
spazio viene riservato anche ai laici, sebbene (dato il loro numero) in percentuale bassa. $\mathrm{Ma}$, più in generale, il Liber mette in scena le orazioni come momenti centrali dell'evoluzione della guerra balearica, snodi fondamentali per capire come fossero andate le cose. Si tratta di un punto sul quale occorre insistere per capire la centralità delle pratiche oratorie nel Liber Maiorichinus. Nello spazio di una generazione - quello che correva tra i protagonisti della guerra di al-Mahdia e la stesura del Liber Maiorichinus - erano cambiate molte cose. I figli di coloro che avevano partecipato alla guerra africana avevano maturato una nuova coscienza culturale: percorsi di formazione differenti dai loro padri, nei quali poteva avere un ruolo più importante lo studio della retorica, avevano portato a una maggiore centralità dell'oratoria. Allo stesso tempo, il contesto politico richiedeva che nei contesti pubblici la parola avesse uno spazio maggiore. Parlare in pubblico - come abbiamo visto - era sinonimo di governo della società. Si trattava, con ogni probabilità, di un'attività regolamentata da codici non scritti dell'interazione sociale: chi non aveva i requisiti, non poteva parlare in pubblico $\mathrm{o}$, se lo faceva, ciò che diceva non era considerato legittimo, era come un mormorio che non meritava di essere citato. Il non sapere/poter parlare era poi considerato caratteristico di una sfera sociale bassa, come se l'educazione alla parola fosse, in un certo senso, un carattere distintivo.

5. Arriviamo così alle conclusioni per qualche considerazione di carattere più generale. Nel Liber Maiorichinus si registra un notevole interesse per le orazioni tenute da illustri esponenti della città, laici ed ecclesiastici. Ci si riferisce alle orazioni con termini differenti, di origine documentaria («querela») o con un'aggettivazione che lascia intendere l'esistenza di tipologie retoriche diversificate a seconda dei contesti e degli interlocutori. Tutto ciò, potrebbe essere spia di una prima formalizzazione delle pratiche oratorie nella Pisa dei primi decenni del XII secolo; allo stesso tempo, quanto detto ci consente di ipotizzare con ragionevole certezza che già da qualche tempo prima della stesura del Liber parlare in pubblico rientrasse tra gli elementi di una nuova cultura politica delle élite cittadine. Diversamente da fasi più tarde della storia comunale, non abbiamo per questo periodo testimonianze dirette di questa formalizzazione. Tuttavia, si può pensare che ci fosse una tendenza in questo senso. Il Liber Maiorichinus fotografa una situazione in movimento da tempo.

Per questa ragione, è da considerare con attenzione il fatto che il Liber rifletta uno stadio relativamente avanzato di questa formalizzazione. Alcuni indizi inducono a riconoscere una vera e propria "fase di accelerazione" di questo processo attorno agli anni di stesura del Liber: proprio attorno alla metà degli anni '20 sono collocabili due fonti dalle quali emerge con chiarezza l'attenzione dei ceti dirigenti alla parola pubblica. La prima è il trattato tra Pisa e Amalfi del 1126, nel quale Timino (il figlio del re sconfitto ad al-Mahdia dai Pisani nel 1087) figura come «publicus preco Pisane civitatis, splendidissime ad vocem totius Populi», cosa che lascia intendere la nomina comunale di un "ufficiale" addetto alla lettura pubblica dei documenti; ${ }^{16}$ la seconda è un frammento cronachistico edito da Pertz col titolo di Notae Pisanae, che custodisce la notizia della guerra tra Pisa e Lucca del 1127 per il castello Aghinolfi, in occasione della quale fu pronunciato un discorso da un anonimo console che la fonte riporta nella forma del discorso diretto. Si tratta, se non consideriamo il Liber Maiorichinus, della prima orazione di un console nella produzione documentaria di Pisa. ${ }^{17}$

In altre parole, sembra proprio che gli anni '20 vadano considerati una soglia decisiva per l'affermazione di una nuova cultura retorica delle élite cittadine. Quali sono le ragioni dell'accelerazione dei processi di formalizzazione in questo periodo? 
Abbiamo visto che, chi con le sue orazioni occupava lo spazio pubblico, aveva una posizione di spicco e abbiamo mostrato, così, che esisteva una forte correlazione tra parola e potere. A parlare sono soprattutto gli esponenti illustri della città: il vescovo, i canonici e i consoli. Non sembra che siano individuabili particolari distinzioni formali tra i discorsi di questi differenti gruppi politico-istituzionali - sebbene l'autore del Liber non manchi di rilevare con variazioni stilistiche di carattere ritmico la peculiarità dell'arte oratoria del vescovo in un'occasione. Abbiamo già rilevato quanto sia difficile capire quanto le orazioni siano specchio della realtà: il Liber Maiorichinus è una testimonianza significativa del processo che portò all'affermazione dell'oratoria nella cultura politica delle élite cittadine a Pisa nel XII secolo. Benché i discorsi riportati siano con ogni probabilità fittizi, rielaborazioni postume, il loro contenuto di verità può essere individuato proprio nella rilevanza data alla pratica dell'oratoria in contesti pubblici, come suggerito da un confronto con le fonti narrative precedenti.

Esiste, forse, un rapporto tra autocoscienza politica e sviluppo dell'eloquenza delle élite cittadine? Da questo punto di vista, non è forse inutile rilevare che, negli stessi anni in cui registriamo questi fenomeni, è collocabile con sicurezza l'attestazione della nuova denominazione del luogo di riunione dell'assemblea cittadina che supera l'assetto politico "marchionale" a cui rimandava la vecchia formula: la «curia» presso la chiesa di San Donato, definita nel 1111 «forum Pisane civitatis, quod curia marchionis appellatur», in un documento del 1126 viene definita «curia Pisana, in communi colloquio, prope ecclesiam sancti Donati», riflesso di un nuovo assetto politicoistituzionale. ${ }^{18}$ Tutto ciò successe all' indomani della morte di Enrico V (1125): come noto, l'imperatore e i marchesi da lui installati alla guida della Marca di Tuscia non ebbero rapporti pacifici con Pisa, che preferì coltivare soprattutto i rapporti con i Pontefici. ${ }^{19}$ Senza voler stabilire una correlazione diretta tra eventi politico-istituzionali e fenomeni culturali, non possiamo non osservare come gli anni immediatamente successivi alla morte dell'imperatore segnarono un tornante decisivo nei processi di formalizzazione della nuova cultura retorica delle città. Si aprì - ancora più marcatamente che nel recente passato - un vuoto di potere, che le élite cittadine tentarono di colmare occupando lo spazio pubblico con la parola. Era un modo per dimostrare di saper governare. Il Liber Maiorichinus, maturato in quegli anni, riflette appieno questa tendenza.

Da tempo, i «cives» si esercitavano nelle orazioni: nei placiti, nei «colloqui civitatis», in tutte quelle occasioni cittadine nelle quali era necessario esprimere la propria opinione. Ma ciò che abbiamo rilevato in queste brevi pagine a partire dal Liber Maiorichinus va oltre il semplice esercizio della parola: si tratta di fare dell'arte della parola un codice culturale, ciò che fino ad allora non era stato. Era uno dei modi attraverso i quali fu costruita la nuova politica nella Pisa del XII secolo.

\footnotetext{
${ }^{1}$ Liber Maiorichinus de gestis Pisanorum illustribus, a cura di G. Scalia, Roma, 2017.

${ }^{2}$ Sulla datazione del testo, a M. Ronzani, A novecento anni dalla vittoria di Maiorca, Pisa, 2015. Più in generale, sul Liber mi permetto di rimandare a A. Cotza, Prove di memoria. Origini e funzioni della storiografia nella Toscana pienomedievale, Roma, 2020 [in corso di stampa], cap. 2.2.

${ }^{3}$ Liber Maiorichinus, pp. 370-375.

${ }^{4}$ Si veda, a titolo d'esempio, Ivi, pp. 210-211: «Ut portum tenuere rates et cetus haberi cepit et herboso steterat iam contio prato»; pp. 274-275: «Tum cetum tenuere patres [...]».

${ }^{5} \mathrm{Su}$ questa tipologia documentaria si veda P. Cammarosano, Carte di querela nell'Italia dei secoli $X$ $X I I I$, in «Frühmittelalterliche Studien», 36, pp. 397-402.
} 
${ }^{6}$ La scena è descritta in Liber Maiorichinus, pp. 212-214.

${ }^{7}$ Ivi, pp. 220-221.

${ }^{8} I v i$, pp. $230-231$

${ }^{9}$ Ivi, pp. 210-213.

${ }^{10}$ Fanno eccezione i primi cinque versi del discorso di Pietro, esametri non caudati, ma comunque con rima o assonanza bisillabica. Si veda, a questo proposito, Liber Maiorichinus, pp. 50-51 e n. 172.

${ }^{11}$ Per le famiglie pisane, si vedano i seguenti saggi. Per gli Orlandi, M. Ronzani, Ranieri, Benincasa e il Barbarossa. Peripezie di un culto nella Pisa dei secoli XII-XIV, Pisa, 2016, pp. 73-91; Marignani: G. Garzella, Marignani, Azzi, Alabarba, in Pisa nei secoli XI e XII: formazione e caratteri di una classe di governo, a cura di G. Rossetti, Pisa, 1979, pp. 65-124; Sismondi: M. Ronzani, La «casa di Gontolino». Origine, sviluppo genealogico e attività pubblica della famiglia dei Sismondi fino ai primi decenni del Duecento, in «Bollettino Storico Pisano», 75, pp. 503-522; De Curte: B. Rovai, La famiglia De Curte tra l'XI e il XIV secolo, tesi di laurea, rel. M. L. Ceccarelli Lemut, Università di Pisa, 1994; Casapieri: L. Ticciati, Strategie familiari della progenie di Ildeberto Albizo - $i$ Casapieri - nelle vicende e nella realtà pisana fino alla fine del XIII secolo, in Pisa e la Toscana occidentale nel medioevo. A Cinzio Violante nei suoi 70 anni, Pisa, 1991, pp. 49-150.

${ }^{12}$ M. C. Rossi, Scritture e scriventi in una città mediterranea. Pisa tra XI e XII secolo, Pisa, 2013, in part. pp. 112-116.

${ }_{13}^{13}$ Liber Maiorichinus, pp. 308-309.

${ }^{14}$ Edita in G. Scalia, Il carme pisano sull'impresa contro i Saraceni del 1087, in Studi di filologia romanza scritti in onore di Silvio Pellegrini, Padova, 1971, pp. 565-627. Si veda H. E. J. Cowdrey, The Mahdia Campaign of 1087, in «The English Historical Review», 92, pp. 1-29; mi permetto di rimandare anche a A. Cotza, Storia, memoria, politica alla fine del secolo XI: il carme pisano sull'impresa contro $i$ saraceni del 1087, in «Archivio Storico Italiano», 175, pp. 37-72.

${ }^{15}$ Gesta Triumphalia per Pisanos facta, a cura di G. Scalia, Firenze, 2010; su cui anche M. Ronzani, A proposito della nuova edizione dei Gesta Triumphalia per Pisanos facta, in «Archivio Storico Italiano», 169 , pp. 373-387.

${ }^{16}$ F. Bonaini, Due carte pisano-amalfitane dei secoli XII e XIV, in «Archivio Storico Italiano», 8, pp. 3-8.

${ }^{17}$ Notae Pisanae, a cura di K. Pertz, in MGH, SS, 19, p. 266.

${ }^{18}$ G. Garzella, I palazzi pubblici a Pisa nel medioevo come specchio dell'evoluzione politico-istituzionale e delle vicende urbanistiche, in Les palais dans la ville. Espaces urbains et lieux de la puissance publique dans la Méditerranée médiévale, a cura di Patrick Boucheron e Jacques Chiffoleau, Lyon, pp. 109-122.

${ }^{19}$ Per un quadro della situazione politica interna ed esterna di Pisa in questo periodo, si veda M. Ronzani, L'affermazione dei Comuni cittadini fra Imperoe Papato : Pisa e Lucca da Enrico IV al Barbarossa (1081-1162), in Poteri centrali e autonomie nella Toscana medievale e moderna, a cura di G. Pinto, L. Tanzini, Firenze, 2012, pp. 1-57. 